九州大学学術情報リポジトリ

Kyushu University Institutional Repository

\title{
Writing and reading of an arbitrary optical polarization state in an antiferromagnet
}

\section{Satoh, Takuya}

Institute of Industrial Science, The University of Tokyo | Department of Physics, Kyushu University | PRESTO, Japan Science and Technology Agency

\section{Iida, Ryugo}

Institute of Industrial Science, The University of Tokyo,

Higuchi, Takuya

Lehrstuhl für Laserphysik, Friedrich-Alexander-Universität Erlangen-Nürnberg

Fiebig, Manfred

Department of Materials, ETH Zurich

他

http://hdl. handle. net/2324/1808892

出版情報 : Nature Photonics. 9 (1)，pp.25-29，2014-11-17. Nature Pub. Group バージョン：

権利関係 : 


\section{Writing and reading of an arbitrary optical polarization state in an antiferromagnet}

Takuya Satoh ${ }^{1,2,3 \# *}$, Ryugo Iida ${ }^{1 \#}$, Takuya Higuchi ${ }^{4}$, Manfred Fiebig $^{5} \&$ Tsutomu Shimura ${ }^{1}$

${ }^{1}$ Institute of Industrial Science, The University of Tokyo, Tokyo 153-8505, Japan

${ }^{2}$ Department of Physics, Kyushu University, Fukuoka 812-8581, Japan

${ }^{3}$ PRESTO, Japan Science and Technology Agency, Tokyo 102-0076, Japan

${ }^{4}$ Lehrstuhl für Laserphysik, Friedrich-Alexander-Universität Erlangen-Nürnberg, 91058 Erlangen,

Germany

${ }^{5}$ Department of Materials, ETH Zurich, 8093 Zurich, Switzerland

\# These authors contributed equally to this work

*e-mail: satoh@phys.kyushu-u.ac.jp

The interaction between light and magnetism is considered a promising route to the development of energy-efficient data storage technologies. To date, However, ultrafast optical magnetization control has been limited to a binary process, whereby light in either of two polarization states generates (writes) or adopts (reads) a magnetic bit carrying either a positive or negative magnetization. Here, we report how the fundamental limitation of just two states can be overcome, allowing an arbitrary optical polarization state to be written magnetically. The effect is demonstrated using a three-sublattice antiferromagnet-hexagonal $\mathrm{YMnO}_{3}$. Its three magnetic oscillation eigenmodes are selectively excited by the three polarization eigenstates of the light. The magnetic oscillation state is then transferred back into 
the polarization state of an optical probe pulse, thus completing an arbitrary optomagnonic write-read cycle.

Two types of interaction between light and magnetism are currently of interest. In the first, the transfer of photon energy to electrons by absorption initiates an interplay of electrons, spins and lattice ions that leads to magnetization changes on timescales down to the femtosecond range ${ }^{1-3}$ and may even involve phase transitions ${ }^{4,5}$. Of particular interest for data storage is all-optical magnetization reversal, as observed in a two-sublattice magnetic metal, where thermally activated ultrafast spin waves have been found to play an important role ${ }^{6}$. Optical excitation can also generate coherent spin waves ${ }^{3,7,8}$, which may enter the realm of magnonics ${ }^{9}$.

In the second type of interaction, optically stimulated magnetization changes occur, with negligible energy transfer by absorption. Coherent magnetization control via Raman scattering ${ }^{10-19}$ in particular, is attracting attention. Typical examples are the inverse Faraday effect (inverse FE) ${ }^{10}$ and the inverse Cotton-Mouton effect (inverse CME) ${ }^{12}$, in which circularly and linearly polarized light pulses, respectively, induce an effective magnetic field in a magnetically ordered medium ${ }^{3}$. Coherent processes of this type have a number of potential applications, including information processing, for example, by transferring the optical polarization state into the magnetization of a material.

A fully polarized light beam possesses three polarization degrees of freedom: two mutually orthogonal directions of oscillation and the phase between these two components. This is parameterized by three Stokes parameters $\left(S_{1,2,3}\right)$ on the Poincaré sphere (Fig. 1a) ${ }^{20}$. Assum- 
ing normalization by the light intensity, these are defined as $S_{1}=\cos 2 \theta, S_{2}=\sin 2 \theta \cos \psi$, and $S_{3}=\sin 2 \theta \sin \psi$, where $\theta$ and $\psi$ are the angles parameterizing the complex amplitudes of the light $\left(\mathscr{E}_{x}=\mathscr{E}_{0} \cos \theta, \mathscr{E}_{y}=\mathscr{E}_{0} \sin \theta \mathrm{e}^{i \psi}\right)$. Binary light-matter information transfer so far uses only two of this infinite number of photonic states. Light with, for example, $S_{3}= \pm 1$ generates a positive or negative magnetization bit. This fundamental limitation is overcome by employing all three degrees of freedom of optical polarization as a non-binary information carrier and representing this state magnetically. However, a one-to-one mapping of the three Stokes parameters onto the magnetic oscillation modes has not as yet been reported. A limitation to such a transfer is that three magnetic sublattices are required for encoding the three Stokes parameters. This may be achieved with a material having multiple magnetic lattice sites or with a three-sublattice antiferromagnet. So far, coherent control of magnetic oscillation with an optical pulse has been demonstrated in compounds with at most two magnetic degrees of freedom ${ }^{3}$. An interesting attempt to emulate three magnetic degrees of freedom with a two-sublattice antiferromagnet has been demonstrated for the antiferromagnetic $\mathrm{NiO}$ by using multidomain samples. In that approach, pairs of linearly polarized light pulses created magnetic oscillations on a circular trajectory ${ }^{18}$. However, excitation with circularly polarized light $\left(S_{3} \neq 0\right)$ was not discussed, and one-to-one mapping between optical and magnetic waves was not anticipated.

In this letter, we demonstrate the transfer between an arbitrary optical polarization state and the magnetic oscillations of the three-sublattice hexagonal antiferromagnet $\mathrm{YMnO}_{3}$. We demonstrate that pump (write) pulses with Stokes parameters $S_{1}, S_{2}$ and $S_{3}$ trigger (ferro)magnetic oscillations along the $x, y$ and $z$ axes, respectively. We also show that the induced magnetization changes 
the Stokes vector $\mathbf{S}^{\prime}$ of a time-delayed probe (read) pulse by $\Delta \mathbf{S}^{\prime}$, with a unique correspondence to the original Stokes vector $\mathbf{S}$. In the final step we demonstrate the linearity of the information transfer. Orthogonal polarization states of two pulses and the associated magnetic oscillations add up in a vector-like manner, without crosstalk.

As shown in Fig. $1 b$, below the Néel temperature $T_{\mathrm{N}} \approx 70 \mathrm{~K}$, hexagonal $\mathrm{YMnO}_{3}$ is antiferromagnetic, with three sublattices carrying equivalent magnetizations $\mathbf{M}_{1}=M_{0}(-1 / 2, \sqrt{3} / 2$, $0), \mathbf{M}_{2}=M_{0}(-1 / 2,-\sqrt{3} / 2,0)$ and $\mathbf{M}_{3}=M_{0}(1,0,0)$, corresponding to spins pointing along the local $x$ axes $^{21}$. This structure was established after initial controversial discussions, as reviewed in Ref. 18. The magnetic oscillations in $\mathrm{YMnO}_{3}$ have one out-of-plane and two in-plane modes, as shown in Fig. 1c. The frequencies of the in-plane ' $\mathrm{X}$ ' and ' $\mathrm{Y}$ ' modes are degenerate, with $\Omega_{\mathrm{X}} / 2 \pi=\Omega_{\mathrm{Y}} / 2 \pi=1.3 \mathrm{THz}^{22-25}$. The frequency of the out-of-plane ' $\mathrm{Z}$ ' mode is $\Omega_{\mathrm{Z}} / 2 \pi=56 \mathrm{GHz}^{24}$. We introduce the ferromagnetic vector $\mathbf{M} \equiv \frac{1}{\sqrt{3}}\left(\mathbf{M}_{1}+\mathbf{M}_{2}+\mathbf{M}_{3}\right)$, where $\mathbf{M}=0$ in the equilibrium state. Then $\mathbf{m}$ is defined as the modulation from this equilibrium state. As shown in Fig. 1c (complemented by the calculation provided in Supplementary Section 1), the $\mathrm{X}, \mathrm{Y}$ and $\mathrm{Z}$ modes correspond to the non-zero components $m_{x}, m_{y}$ and $m_{z}$, respectively. They can be selectively probed by the three configurations shown in Fig. 2 (X-, Y- and Z-probes; see Methods). Note that the $\mathrm{YMnO}_{3}(0001)$ plane has three-fold symmetry and is optically isotropic for light incident perpendicular to the (0001) plane, so polarization is preserved in the sample.

In the experiment (see Methods), an $\mathrm{YMnO}_{3}(0001)$ single crystal was pumped optically to transfer the polarization state of light $\mathbf{S}$ into magnetic oscillation $\mathbf{m}$. A time-delayed probe pulse 
is modulated by this magnetic oscillation by the CME and FE to transfer it back into an optical polarization state uniquely related to $\mathbf{S}$. This process is summarized in Fig. 1a and is complemented by Supplementary Movie 1. To verify the one-to-one correspondence of the twofold transfer, we first have to identify the magnetic oscillation modes and establish the relation between $S_{1,2,3}$ and $m_{x, y, z}$. We therefore excited the $\mathrm{YMnO}_{3}$ sample with a pump pulse using $\left(S_{1}, S_{2}, S_{3}\right)=(1,0,0)$ and employed the X-probe configuration. Figure 3a presents the raw data of the measured modulation of the probe pulse, which is a superposition of various oscillations. Figure $3 b$ shows their spectrum, obtained by Fourier transform (see Methods). The peak at $1.3 \mathrm{THz}$ with a width of $0.2 \mathrm{THz}$ is a magnetic mode ${ }^{22-25}$, whereas peaks at 4.2, 7.0, 7.5 and 9.2 $\mathrm{THz}$ are $E_{2}$ phonon modes ${ }^{25-27}$. As expected, the $\mathrm{Z}$ mode was not observed because $S_{3}=0$ and thus $m_{z}=0$. To verify the distinction between the degenerate $\mathrm{X}$ and $\mathrm{Y}$ modes, we pumped the sample with light in polarization states $S_{1}= \pm 1$ or $S_{2}= \pm 1$ and employed the X-and Y-probe configurations. Figure $3 \mathrm{c}, \mathrm{d}$ present the time dependence of the corresponding signals derived from measured data, as in Fig. 3a, by extracting the frequency component at $1.3 \pm 0.1 \mathrm{THz}$ by Fourier transform (Supplementary Movie 2). The correspondence is striking; with only a small, alignment-related background caused by an angle of $<1^{\circ}$ between the wavevector of the light and the (0001) axis of the crystal, pump pulses with $S_{1}= \pm 1$ or $S_{2}= \pm 1$ excite the magnetic oscillations $\mp m_{x}$ and $\pm m_{y}$, respectively. Furthermore, as seen in Fig. 3e, when the linear polarization (that is, $\psi \equiv 0$ ) is varied by tuning $\theta$, the signed amplitude of the $1.3 \mathrm{THz}$ component is well fitted by the relation $\cos 2 \theta\left(\propto S_{1}\right)$ and $\sin 2 \theta\left(\propto S_{2}\right)$ in the X-and Y-probe configurations, respectively.

Finally, the $\mathrm{YMnO}_{3}$ sample was pumped with circularly polarized light, $S_{3}= \pm 1$, and the Z- 
probe configuration was used. In this case, the $\mathrm{Z}$ mode with $56 \mathrm{GHz}$ was observed, as shown in Fig. 3f, whereas none of the magnon or phonon modes in Fig. $3 \mathrm{~b}$ can be excited by circularly polarized light (for derivation see Supplementary Sections 1 and 3). In addition, no magnetic mode was detected in the Z-probe configuration when the pump pulses were linearly polarized $\left(S_{3}=0\right)$, and no magnetic mode was detected in the X- or Y-probe configurations when the pump pulses were circularly polarized $\left(S_{1}=S_{2}=0\right)$. X, Y and Z modes disappear above $T_{\mathrm{N}}$, reflecting their magnetic origin. All this ultimately confirms the one-to-one nature of the pump light $\rightarrow$ magnetization $\rightarrow$ probe light correspondence.

We now consider the theoretical background of how the optical pump pulse triggers the magnetic oscillation in $\mathrm{YMnO}_{3}$ (for details see Supplementary Section 1). The pump pulse generates an impulsive effective magnetic field pulse $\mathbf{H}_{\mathrm{eff}, \alpha}$ via the inverse CME and inverse $\mathrm{FE}^{12}$, where $\alpha \in\{1,2,3\}$ is the sublattice number (according to Fig. 1b). The pulse duration and the effective magnetic field $\mathbf{H}_{\mathrm{eff}, \alpha}$ are described with a delta function $\delta(t)$ because it is substantially shorter than $2 \pi / \Omega_{\mathrm{X}, \mathrm{Y}, \mathrm{Z}}$. The magnetization dynamics are described by the Landau-Lifshitz equation $\mathrm{d} \mathbf{M}_{\alpha} / \mathrm{d} t=-\gamma \mathbf{M}_{\alpha} \times\left(\mathbf{H}_{\mathrm{eff}, \alpha}+\mathbf{H}_{\mathrm{E}, \alpha}+\mathbf{H}_{\mathrm{A}, \alpha}+\mathbf{H}_{\mathrm{A}^{\prime}, \alpha}\right)$, where $\mathbf{H}_{\mathrm{E}, \alpha}, \mathbf{H}_{\mathrm{A}, \alpha}$, and $\mathbf{H}_{\mathrm{A}^{\prime}, \alpha}$ represent exchange, out-of-plane anisotropy and in-plane anisotropy fields, respectively, and $\gamma>0$ is the gyromagnetic constant. When the coordinates of the magnetic oscillations $Q_{\mathrm{X}}, Q_{\mathrm{Y}}$ and $Q_{\mathrm{Z}}$ are introduced, the Hamiltonian of the magnetic oscillation becomes diagonal: $\mathscr{H}_{\text {oscillation }}=\sum_{n}\left|Q_{n}\right|^{2} \Omega_{n}$ $(n \in\{\mathrm{X}, \mathrm{Y}, \mathrm{Z}\})$, with $\Omega_{\mathrm{X}}=\Omega_{\mathrm{Y}}=\gamma \sqrt{H_{\mathrm{A}}\left(3 H_{\mathrm{E}} / 2+H_{\mathrm{A}^{\prime}}\right)}$ and $\Omega_{\mathrm{Z}}=\gamma \sqrt{H_{\mathrm{A}^{\prime}}\left(3 H_{\mathrm{E}}+H_{\mathrm{A}}\right)^{24}}$. Expressing the coordinates of the magnetic oscillation in terms of the Stokes parameters leads to $Q_{\mathrm{X}} \propto S_{1} e^{i \Omega_{\mathrm{X}} t}, Q_{\mathrm{Y}} \propto S_{2} e^{i \Omega_{\mathrm{Y}} t}, Q_{\mathrm{Z}} \propto S_{3} e^{i \Omega_{\mathrm{Z}} t}$ (Supplementary equations (S13)-(S15)). Thus, the ex- 
perimentally verified one-to-one mapping $S_{1} \rightarrow Q_{\mathrm{X}}, S_{2} \rightarrow Q_{\mathrm{Y}}, S_{3} \rightarrow Q_{\mathrm{Z}}$ is also established by theory.

We next scrutinize the interaction between the magnetic medium and the probe pulse. Transforming the magneto-optical contributions by the $\mathrm{CME}$ and $\mathrm{FE}$ into a variation $\delta \varepsilon_{i j}^{\prime}$ of the dielectric function (for explicit calculation see Supplementary Section 2) leads to $\operatorname{Re}\left[Q_{\mathrm{X}}\right] \propto \delta \varepsilon_{x x}^{s^{\prime}}=-\delta \varepsilon_{y y}^{s^{\prime}}$, $\operatorname{Re}\left[Q_{\mathrm{Y}}\right] \propto \delta \varepsilon_{x y}^{s^{\prime}}$ and $\operatorname{Re}\left[Q_{\mathrm{Z}}\right] \propto \delta \varepsilon_{x y}^{a^{\prime}}$, where $s$ and $a$ denote symmetric $\left(\delta \varepsilon_{i j}^{s^{\prime}}=\delta \varepsilon_{j i}^{s^{\prime}}\right)$ and antisymmetric $\left(\delta \varepsilon_{i j}^{a^{\prime}}=-\delta \varepsilon_{j i}^{a^{\prime}}\right)$ contributions to $\delta \varepsilon_{i j}^{\prime}$, respectively. This corresponds to a one-to-one mapping $\mathbf{Q} \rightarrow \Delta \mathbf{S}^{\prime} ; \Delta \mathbf{S}^{\prime} \propto \mathbf{S}^{\prime} \times(\hat{\mathrm{A}} \cdot \operatorname{Re}[\mathbf{Q}])$, where $\hat{\mathrm{A}}$ is a diagonal matrix of constants (Supplementary equation (S21)). $\Delta \mathbf{S}^{\prime}$ denotes a modulation of the probe Stokes vector $\mathbf{S}^{\prime}$. The geometry of the $\mathrm{X}-$, Yand Z-probe configurations in Fig. 2 is chosen such that the $\delta \varepsilon_{i j}^{\prime}$ modulation corresponding to the respective mode $Q_{\mathrm{X}}, Q_{\mathrm{Y}}$ or $Q_{\mathrm{Z}}$ and, further, to the Stokes parameters $S_{1}, S_{2}$ or $S_{3}$ of the original pump pulse, is filtered out.

In the final step we investigate the linearity of the light-matter interaction processes discussed here. So far we have only considered the transfer of unmixed photonic states, that is, light polarized along one of the principal axes of the Poincaré sphere and a single magnetic oscillation mode. For an arbitrary polarization state we have to verify that there are no higher-order (nonlinear) effects coupling different magnetic oscillation modes among one another or to the pump or probe light field 28,29 . This verification is acquired with two consecutive pump pulses in polarization states $S_{1}=-1$ and $S_{2}=+1$. Their timing is chosen such that the vector-like addition of the two excitations yields a circular or linear magnetic oscillation state. Any of the aforementioned higher- 
order effects would destroy this oscillation state upon propagation and convert the circular or linear magnetic oscillation into an elliptical one.

Figure 4 shows the result of double-pulse excitations in which the first pulse was incident at $t=0$ and the second one after $0.6,0.8$ or $1.0 \mathrm{ps}$ (that is, $3 / 4,1$ or $5 / 4$ periods of the magnetic oscillation, respectively). This yields, respectively, anticlockwise, diagonal, and clockwise trajectories of the magnetic oscillation (Supplementary Movie 3). Neither state deforms upon propagation, which reveals that none of the higher-order effects is present. This demonstrates that the light $\rightarrow$ magnetism $\rightarrow$ light one-to-one mapping is indeed applicable to arbitrary polarization states. Note that the damping factor of less than 0.03 is consistent with previously obtained values ${ }^{22,23,25}$, which should be low enough for ultrafast operation using femtosecond light pulses.

In summary, we have demonstrated the one-to-one transfer of the polarization eigenstates of a fully polarized pump light wave onto the magnetic eigenmodes of a three-sublattice antiferromagnet. We converted the magnetic information back into the optical polarization eigenstate of a probe light wave in an equally one-to-one process. Finally, we have demonstrated the linearity of this light $\rightarrow$ magnetism $\rightarrow$ light transfer of information. The aspect of spin-wave engineering relates our work to the emerging field of magnonics ${ }^{9}$. In magnonics, the generation, propagation and detection of spin waves in ferromagnetic systems are investigated. A sub-field of magnonics even discusses the optical generation of spin waves in ferromagnets and their dynamics $3,7,8,30$. This similarity, however, is misleading. Our work discusses the excitation of spin waves in an antiferromagnetic system. The focus is placed on the generation of a spin wave as a non-binary 
information carrier rather than on the manipulation of the spin wave propagation. Here, the orders of magnitude higher eigenfrequencies of antiferromagnets in comparison to ferromagnets and the linearity of our process are important aspects for performing logical operations. Because the information transfer employs the entire three-dimensional 'bandwidth' of an optical polarization state, it can be regarded as a write/read cycle for non-binary quantum information. As a conclusion, one might say that our work builds a one-to-one bridge between optics and magnonics with which we promote the field of 'optomagnonics'.

\section{Methods}

Figure 2a presents the optical pump-probe transmission set-up used here. An amplified Ti:sapphire laser with a centre wavelength of $800 \mathrm{~nm}$, a pulse width of $50 \mathrm{fs}$ and a repetition rate of $1 \mathrm{kHz}$ was used as the primary light source. Part of this light was converted to a wavelength of 1,270 $\mathrm{nm}$ in an optical parametric amplifier, and this was used to pump a flux-grown polished $\mathrm{YMnO}_{3}(0001)$ single-crystal platelet with a thickness of $30 \mu \mathrm{m}$, with a laser fluence of $13 \mathrm{~mJ} / \mathrm{cm}^{2}$. The other part of the fundamental light passed through a delay line before entering the sample as a time-delayed probe pulse. The spot diameters of pump and probe pulses on the sample surface were $100 \mu \mathrm{m}$ and $30 \mu \mathrm{m}$, respectively.

The polarization state of the probe pulse was tailored, as depicted in Fig. 2b, for detecting the respective eigenmodes of the magnetic oscillations in $\mathrm{YMnO}_{3}$. The $\mathrm{X}$ mode modulates $\delta \varepsilon_{x x}^{s^{\prime}}=$ $-\delta \varepsilon_{y y}^{s^{\prime}}$. This was probed via the CME with a circularly polarized incident probe beam. After passing through the sample, the difference between the intensities of the $45^{\circ}$ - and $135^{\circ}$-linearly 
polarized components was measured. The Y mode modulates $\delta \varepsilon_{x y}^{s^{\prime}}$. This was also probed via the CME and with circularly polarized incident light. After passing through the sample, the difference between the intensities of the $0^{\circ}$ - and $90^{\circ}$-linearly polarized components was measured. The $\mathrm{Z}$ mode modulates $\delta \varepsilon_{x y}^{a^{\prime}}$. This was probed via the FE and with incident light polarized linearly at $45^{\circ}$. After passing through the sample, its rotation was analyzed by comparing the components polarized at $0^{\circ}$ and $90^{\circ}$. Division of the transmitted probe pulse into two orthogonally polarized components was achieved with a Wollaston prism.

The temporal waveforms of the probe modulations as functions of the pump-probe delay (Fig. 3a) were Fourier-transformed into spectra in the frequency domain (Fig. 3b). For this transform, a pump-probe delay ranging from 0.8 to 10 ps was chosen. We avoided including the large signal at around $t=0$, to which other non-magnetic nonlinear optical effects, such as two-photon absorption and cross-phase modulation, contribute. The pump-probe delays were chosen so that the magnon and the phonon modes were resolved sufficiently in the frequency domain. Because these modes are already known to be narrowband and well-separated, having three data points in the frequency domain is sufficient to unambiguously identify the presence of the known resonances in $\mathrm{YMnO}_{3}{ }^{22-25}$. This spectral resolution is also sufficient to isolate the temporal waveform of the probe-polarization modulation from other contributions via spectral filtering around a narrowband peak and via the inverse Fourier transform (Fig. 3c,d). On the basis of this estimate, a set of pump-probe delays with a step of $33 \mathrm{fs}$ (which corresponds to a spectral bandwidth of $30 \mathrm{THz}$ ) and a range of $10 \mathrm{ps}$ (that is a spectral resolution of $0.1 \mathrm{THz}$ ) was chosen for the $\mathrm{X}$ - and Y-probe configurations to resolve these modes, including the 1.3-THz X and Y magnon modes. 
The probe signal was integrated over $30 \mathrm{~s}$ for each data point. We limited the number of data points (10 ps/33 fs $\sim 300$ points) to acquire a data set within the time of stable operation of the laser, which is typically several hours.

\section{References}

1. Beaurepaire, E., Merle, J.-C., Daunois, A. \& Bigot, J.-Y. Ultrafast spin dynamics in ferromagnetic nickel. Phys. Rev. Lett. 76, 4250-4253 (1996).

2. Koopmans, B., van Kampen, M., Kohlhepp, J. T. \& de Jonge, W. J. M. Ultrafast magneto-optics in nickel: Magnetism or optics? Phys. Rev. Lett. 85, 844-847 (2000).

3. Kirilyuk, A., Kimel, A. V. \& Rasing, Th. Ultrafast optical manipulation of magnetic order. Rev. Mod. Phys. 82, 2731-2784 (2010).

4. Takubo, N. et al. Persistent and reversible all-optical phase control in a manganite thin film. Phys. Rev. Lett. 95, 017404 (2005).

5. Ju, G. et al. Ultrafast generation of ferromagnetic order via a laser-induced phase transformation in FeRh thin films. Phys. Rev. Lett. 93, 197403 (2004).

6. Ostler, T. A. et al. Ultrafast heating as a sufficient stimulus for magnetization reversal in a ferrimagnet. Nature Commun. 3, 666 (2012).

7. Van Kampen, M. et al. All-optical probe of coherent spin waves. Phys. Rev. Lett. 88, 227201 (2002). 
8. Müller, G. M. et al. Magnetization dynamics in optically excited nanostructured nickel films. New J. Phys. 10, 123004 (2008).

9. Demokritov, S. O. \& Slavin, A. N. Magnonics: From Fundamentals to Applications (Springer, 2013).

10. Kimel, A. V. et al. Ultrafast non-thermal control of magnetization by instantaneous photomagnetic pulses. Nature 435, 655-657 (2005).

11. Hansteen, F., Kimel, A., Kirilyuk, A. \& Rasing, Th. Femtosecond photomagnetic switching of spins in ferrimagnetic garnet films. Phys. Rev. Lett. 95, 047402 (2005).

12. Kalashnikova, A. M. et al. Impulsive generation of coherent magnons by linearly polarized light in the easy-plane antiferromagnet $\mathrm{FeBO}_{3}$. Phys. Rev. Lett. 99, 167205 (2007).

13. Gridnev, V. N. Phenomenological theory for coherent magnon generation through impulsive stimulated Raman scattering. Phys. Rev. B 77, 094426 (2008).

14. Satoh, T. et al. Spin oscillations in antiferromagnetic NiO triggered by circularly polarized light. Phys. Rev. Lett. 105, 077402 (2010).

15. Nishitani, J., Kozuki, K., Nagashima, T. \& Hangyo, M. Terahertz radiation from coherent antiferromagnetic magnons excited by femtosecond laser pulses. Appl. Phys. Lett. 96, 221906 (2010).

16. Iida, R. et al. Spectral dependence of photoinduced spin precession in $\mathrm{DyFeO}_{3}$. Phys. Rev. $B$ 84, 064402 (2011). 
17. Higuchi, T., Kanda, N., Tamaru, H. \& Kuwata-Gonokami, M. Selection rules for lightinduced magnetization of a crystal with threefold symmetry: the case of antiferromagnetic NiO. Phys. Rev. Lett. 106, 047401 (2011).

18. Kanda, N. et al. The vectorial control of magnetization by light. Nature Commun. 2, 362 (2011).

19. Popova, D., Bringer, A. \& Blügel, S. Theoretical investigation of the inverse Faraday effect via a stimulated Raman scattering process. Phys. Rev. B 85, 094419 (2012).

20. Yariv, A. \& Yeh, P. Photonics: Optical Electronics in Modern Communications (Oxford University Press, 2007).

21. Lorenz, B. Hexagonal manganites- $\left(\mathrm{RMnO}_{3}\right)$ : class (I) multiferroics with strong coupling of magnetism and ferroelectricity. ISRN Conden. Matter Phys. 2013, 497073 (2013).

22. Penney, T., Berger. P. \& Kritiyakirana, K. Far-infrared antiferromagnetic resonance in hexagonal $\mathrm{YMnO}_{3}$. J. Appl. Phys. 40, 1234-1235 (1969).

23. Kadlec, C. et al. Terahertz and infrared spectroscopic evidence of phonon-paramagnon coupling in hexagonal piezomagnetic $\mathrm{YMnO}_{3}$. Phys. Rev. B 84, 174120 (2011).

24. Sato, T. J. et al. Unconventional spin fluctuations in the hexagonal antiferromagnet $\mathrm{YMnO}_{3}$. Phys. Rev. B 68, 014432 (2003).

25. Toulouse, C. et al. Lattice and spin excitations in multiferroic $h-\mathrm{YMnO}_{3}$. Phys. Rev. B 89, 094415 (2014). 
26. Iliev, M. N. et al. Raman- and infrared-active phonons in hexagonal $\mathrm{YMnO}_{3}$ : experiment and lattice-dynamical calculations. Phys. Rev. B 56, 2488-2494 (1997).

27. Goian, V. et al. THz and infrared studies of multiferroic hexagonal $\mathrm{Y}_{1-x} \mathrm{Eu}_{x} \mathrm{MnO}_{3}(x=0-0.2)$ ceramics. Phase Trans. 83, 931-941 (2010).

28. Frey, J., Frey, R., Flytzanis, C. \& Triboulet, R. Theoretical and experimental investigation of nonlinear Faraday processes in diluted magnetic semiconductors. J. Opt. Soc. Am. B 9, 132-142 (1992).

29. Zvezdin, A. K. \& Kotov, V. A. Modern Magnetooptics and Magnetooptical Materials (IOP Publishing, 1997).

30. Satoh, T. et al. Directional control of spin-wave emission by spatially shaped light. Nature Photon. 6, 662-666 (2012).

Acknowledgements The authors thank A. M. Kalashnikova, T. J. Sato, and D. Meier for discussions. This work was supported by the Japan Science and Technology Agency (JST) - Precursory Research for Embryonic Science and Technology (PRESTO) (T.Sa).

Author contributions T.Sa. planned the study. R.I. and T.Sa. carried out the experiment. R.I., T.H., and T.Sa. analyzed the data. M.F. contributed to their interpretation. T.Sh supervised the study. All authors discussed the results and wrote the manuscript.

Additional information Supplementary information is available in the online version of the paper. Reprints 
and permission information is available online at http://www.nature.com/reprints. Correspondence and requests for materials should be addressed to T.Sa.

Competing financial interests The authors declare no competing financial interests. 
Figure 1 Writing and reading of an optical polarization state in three-sublattice $\mathrm{YMnO}_{3}$. a, Illustration of one-to-one information transfer light $\rightarrow$ magnetism $\rightarrow$ light in hexagonal $\mathrm{YMnO}_{3}$. One-to-one mapping between the Stokes parameters of the pump pulse and the magnetic oscillation modes of $\mathrm{YMnO}_{3}$, and between these oscillation modes and the Stokes parameters of the probe pulse (prime symbol), is shown. A probe pulse component $S_{i}^{\prime}$ is modified by a magnetic oscillation along the $j$-axis (resulting from a pump pulse component $S_{j}$ ) by an oscillation $\Delta S_{k}^{\prime}$ with $i j k$ according to the Levi-Civita tensor $\varepsilon_{i j k}$. Specific choices for $i j k$ correspond to the $\mathrm{X}$-, Y- and Z-probe configurations as shown here and in Fig. 2b. b. Antiferromagnetic three-sublattice ordering of the magnetic $\mathrm{Mn}^{3+}$ moments. c, Magnetic oscillation eigenmodes $\mathrm{X}, \mathrm{Y}$ and $\mathrm{Z}$.

Figure 2 Schematics of the experimental set-up. a, Experimental configuration. OPA, Optical parametric amplifier; WP, Wollaston prism; $\lambda / 2$, half-wave plate; $\lambda / 4$, quarterwave plate; BS, beamsplitter; M, mirror. Quarter-wave plates indicated by dotted boxes were introduced only for experiments using circularly polarized light. For the double-pulse experiment, the mirror M' was replaced by a beamsplitter in the path for the pump pulse. $\mathbf{b}$, $\mathrm{X}$-, Y- and Z-probe configurations and their relation to the CME and the FE, as discussed in the main text and in the Methods. Blue and red lines represent the polarizations of the probe pulse in the absence or presence of the pump pulse, respectively.

Figure 3 Experiments for scrutinizing the one-to-one nature of information transfer. a, Time-dependent ellipticity exerted by a pump pulse with $S_{1}=+1$ on a probe pulse 
measured in the X-probe configuration. $T=5 \mathrm{~K}$. b. Fast Fourier transform (FFT) spectrum of the data in a. a.u., arbitrary units. The magnetic oscillation mode at $1.3 \mathrm{THz}$ is indicated by a red arrow. c,d, Magnetic 1.3 THz components excited by a pump pulse with $S_{1}= \pm 1$ (c) and $S_{2}= \pm 1$ (d). Data were extracted from measurements as in aby Fourier transform. The X-and Y-probe configurations were applied according to Fig. 2, revealing a one-toone relation $S_{1} \sim \mathrm{X}$ and $S_{2} \sim \mathrm{Y}$. e, Signal detected in the X-probe (green) and Y-probe (blue) configurations with dependence of the angle $\theta$ parameterizing the linear $(\psi \equiv 0)$ polarization of the pump pulse. Lines are proportional to $\cos 2 \theta$ (green) and $\sin 2 \theta$ (blue). f, Time-dependent Faraday rotation exerted on the probe pulse by a circularly polarized pump pulse, $S_{3}= \pm 1 . T=5 \mathrm{~K}$.

Figure 4 Experimental result for double-pulse excitations. a-c, $1.3 \mathrm{THz}$ components excited by a pair of linearly polarized pulses $S_{1}=-1$ and $S_{2}=1$ delayed by $3 / 4$ period (a), 1 period, (b) and 5/4 period (c). X- and Y-probe configurations were used to detect $-m_{x}$ and $m_{y}$, respectively. Data were extracted from measurements, as in Fig. 3a, by Fourier transform. a.u., arbitrary units. $T=5 \mathrm{~K}$. 
a

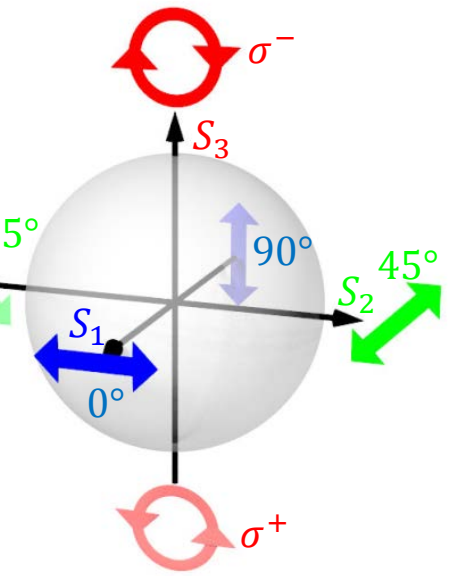

Pump Stokes parameter $\mathbf{S}$

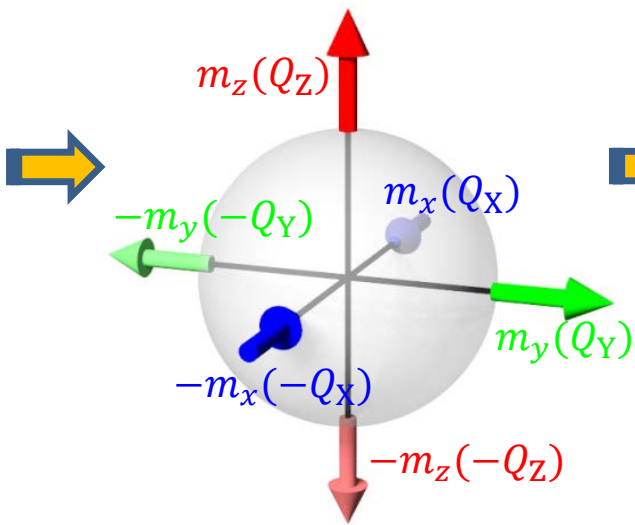

Magnetic oscillation $\mathbf{m}(\mathbf{Q})$

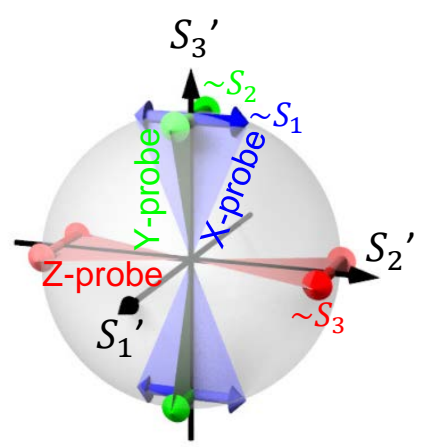

Modulation of probe Stokes parameter $\Delta \mathbf{S}^{\prime}$

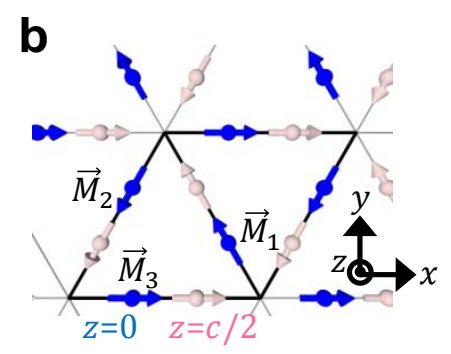

C
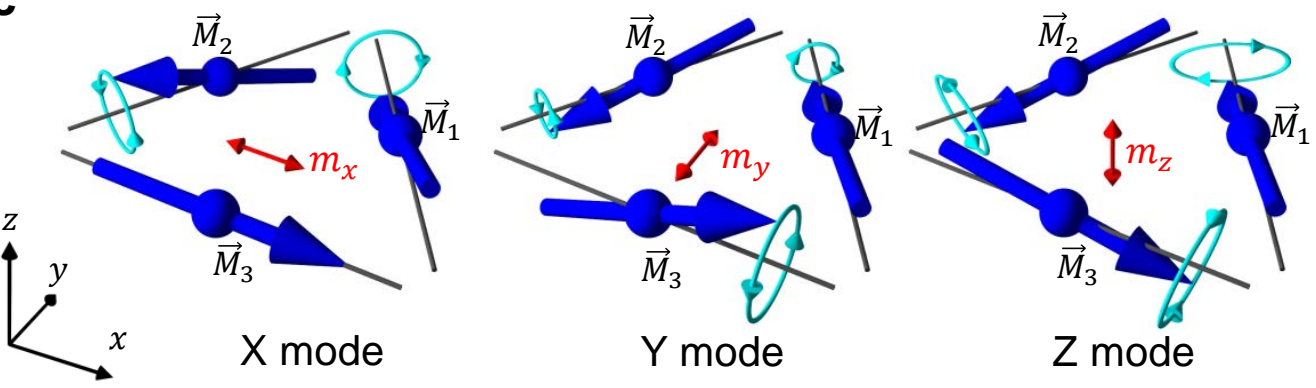

Figure 1 T. Satoh et al., Nature Photonics 
a

Ti: sapphire laser

\& amplifier

M

Delay line

b
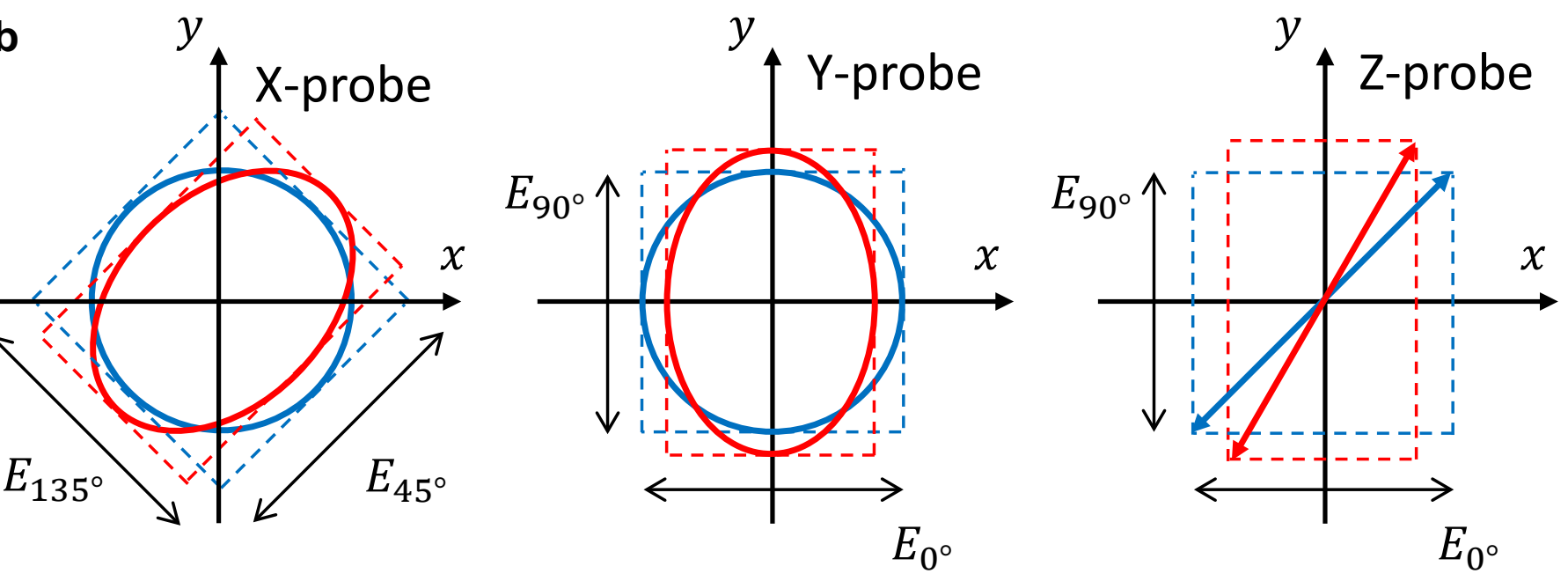

Figure 2 T. Satoh et al., Nature Photonics 
